\title{
Effects of Beta-Lactam Antibiotics on the Acetaldehyde-Metabolizing System in Germ-Free Rats
}

\author{
Takashi MATSUBARA, Shigemi OTSUBO, Ayako OGAWA \\ and Hiroyuki NAKAO* \\ Shionogi Research Laboratories, Shionogi \& Co. Ltd. Fukushima-ku. Osaka 553, Japan \\ *Aburahi Laboratories, Shionogi Research Laboratories, Shionogi \& Co. Ltd.. \\ Koka-cho. Shiga 520-34, Japan \\ Accepted June 1, 1987
}

\begin{abstract}
Effects of several beta-lactam antibiotics on the acetaldehyde-metabolizing system were studied using germ-free rats. Administration of cefamandole (CMD) to the rats caused a decrease in liver mitochondrial low $K_{\mathrm{ra}}$ aldehyde dehydrogenase activity and an increase in blood acetaldehyde level during ethanol metabolism, similar to the case in conventional rats. Oral administration of CMD produced a pronounced increase in blood acetaldehyde level compared to the subcutaneous administration of the antibiotic. When the animals were given various beta-lactam antibiotics subcutaneously, only the antibiotics having an $\mathrm{N}$-methyltetrazolylthiomethyl group at the 3 -position of the cephalosporin nucleus exhibited the disulfiram-like effects on the acetaldehyde-metabolizing system. The results indicate that intestinal bacteria do no participate in the development of the disulfiram-like reaction of several beta-lactam antibiotics.
\end{abstract}

Like disulfiram, several beta-lactam antibiotics, having an $\mathrm{N}$-methyltetrazolylthiomethyl group at the 3 -position of the cephalosporin nucleus, increase blood acetaldehyde level when animals pretreated with these antibiotics were given ethanol $(1-3)$. Subsequent studies demonstrated the depression of hepatic low $K_{m}$ aldehyde dehydrogenase (ALDH) activity following the administration of these antibiotics (4-6). Yanagihara et al. (7) and Turcan et al. (8) demonstrated that biliary excretion of the antibiotics is required for the development of disulfiram-like effects. Recently, we demonstrated that $\mathrm{N}$-methyltetrazolethiol (1methyl-1H-tetrazole-5-thiol, NMTT), a side chain thiol group of the antibiotics, was liberated from the parent antibiotics excreted into the bile (9). Turcan et al. also suggested that gut microfloral mediated degradation of the antibiotics caused the release of NMTT in the body (8). Thus, it should be interesting to determine whether the degradation of these antibiotics by intestinal bacteria is required for the de- velopment of the disulfiram-like effects. We examined the effect of several beta-lactam antibiotics on the acetaldehyde-metabolizing system in germ-free rats to clarify the participation of intestinal bacteria.

\section{Materials and Methods}

The adult male Wistar/Shi strain germfree rats (7 weeks old) used for the experiments were kept in an air-conditioned flexible film isolator $\left(22-25^{\circ} \mathrm{C}, \quad 40-60 \%\right.$ humidity) lighted $12 \mathrm{hr}$ a day (7:00-19:00) and maintained on commercial rat chow (CMF, Oriental Yeast Co. Tokyo) and sterilized water ad libitum. The chow used was irradiated with ${ }^{60} \mathrm{Co}$ of $5 \mathrm{Mrad}$.

Cefamandole (CMD) and moxalactam or latamoxef (LMOX) were obtained from Shionogi \& Co. (Osaka), cefmetazole (CMZ) from Sankyo Co. (Tokyo), cefoperazone (CPZ) from Toyama Chemical Co. (Tokyo). cefazolin (CEZ) from Fujisawa Pharmaceutical Co. (Osaka) and cefotiam (CTM) from Takeda Chemical Industries (Osaka). These antibiotics were dissolved in sterilized 
distilled water at $250 \mathrm{mg} / \mathrm{ml}$, and the resulting solutions were administered at $4 \mathrm{ml} / \mathrm{kg}$ body weight $(1.0 \mathrm{~g}$ antibiotics $/ \mathrm{kg})$ in the isolator. After the administration of antibiotics, the animals were fasted for $18 \mathrm{hr}$ and then orally given a $20 \%(\mathrm{w} / \mathrm{v})$ solution of ethanol in water at $1.0 \mathrm{ml} / 100 \mathrm{~g}$ body weight. Blood and liver samples were obtained $1 \mathrm{hr}$ after ethanol administration, and the hepatic ALDH activity and blood ethanol and acetaldehyde levels were determined as described previously (5).

After the sampling of the blood and liver. a small portion of the caecum contents was inoculated into a thioglycolate-broth medium (Daigo-Eiyo). This was incubated at $37^{\circ} \mathrm{C}$ for 14 days, and then a portion of the culture medium was transferred to either $10 \%$ blood containing heart infusion-agar (Difco) or GAM-agar (Nissui), and incubated further aerobically or anaerobically at $37^{\circ} \mathrm{C}$ for 7 days. Portions of the caecum contents were also inoculated into a culture media of potato dextrose-broth (Difco), potato dextrose-agar (Nissui) and $10 \%$ blood containing agar (Difco), and incubated aerobically at room temperature for 14 days. No bacteria were detected by any of these culture methods in the caecum contents of the germ-free rats used.

\section{Results}

A marked decrease in liver mitochondrial low $\mathrm{K}_{\mathrm{m}} \mathrm{ALDH}$ activity was observed in germfree rats administered CMD either subcutaneously or orally. while the activity of high $K_{m}$ ALDH was not affected by this treatment (Table 1). When ethanol was given to the CMD-pretreated rats, the blood acetaldehyde level increased markedly. A pronounced increase was observed in the blood acetaldehyde level, similar to the case of conventional rats (7). when CMD was given orally. Blood ethanol level also tended to increase in the CMD-pretreated rats.

Next, the germ-free animals were given various beta-lactam antibiotics followed by ethanol, and their ALDH activities and the blood acetaldehyde and ethanol levels were determined. Although liver mitochondrial high $K_{m}$ ALDH activity was not affected by the subcutaneous administration of antibiotics, the low $K_{m}$ enzyme activity decreased in rats treated with either $C M Z, C P Z$ or LMOX: CEZ- or CTM-treated rats showed almost the same activity as that of the control rats (Table 2). The blood acetaldehyde level during ethanol metabolism showed significantly high values in rats showing lower low $K_{m}$ ALDH activity, while the acetaldehyde level in CEZ- and CTMpretreated rats did not differ from that of the control rats. On the other hand, the blood ethanol level in germ-free rats administered various antibiotics tended to increase as in the case of the conventional rats, suggesting

Table 1. Alteration of liver mitochondrial aldehyde dehydrogenase activity and blood acetaldehyde and ethanol levels in germ-free rats following administration of CMD

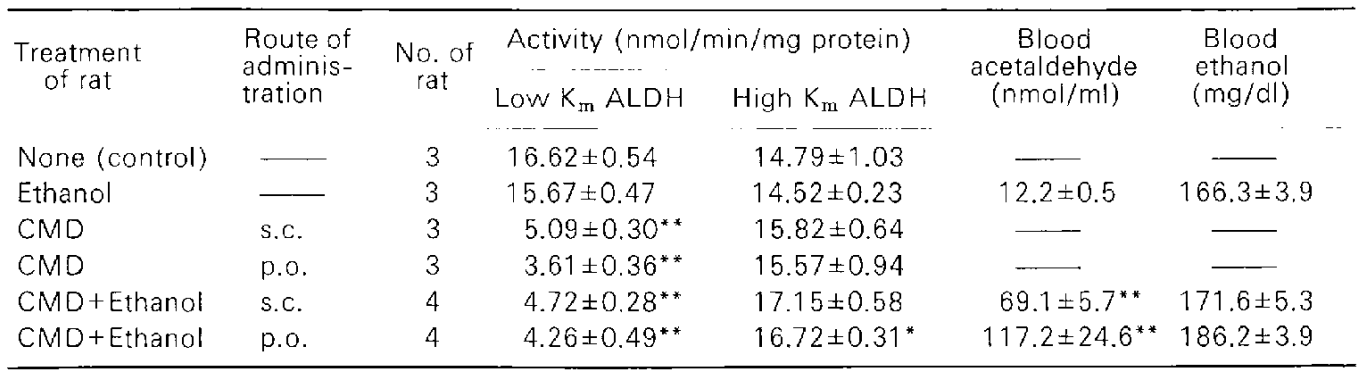

The animals were given CMD subcutaneously or orally at $1.0 \mathrm{~g} / \mathrm{kg}$ and then ethanol $(2.0 \mathrm{~g} / \mathrm{kg}) \mathrm{orally}$ $18 \mathrm{hr}$ after the CMD treatment. Blood and liver samples were obtained $1 \mathrm{hr}$ after the ethanol administration. Animals that received CMD alone were killed $19 \mathrm{hr}$ after the treatment. The values in the table represent the mean \pm S.E. Statistical analyses ("P<0.05 and ** $P<0.01$ ) in ALDH activity and in blood acetaldehyde and ethanol levels were performed against the control and ethanol-treated groups. respectively. 
Table 2. Effects of single administration of various antibiotics on liver mitochondrial ALDH activity and blood acetaldehyde and ethanol levels in germ-free rats

\begin{tabular}{|c|c|c|c|c|c|c|}
\hline \multirow{3}{*}{$\begin{array}{l}\text { Treatment } \\
\text { of rat }\end{array}$} & \multirow{2}{*}{$\begin{array}{l}\text { No. of } \\
\text { rat }\end{array}$} & \multicolumn{3}{|c|}{ Activity (nmol/min/mg protein) } & \multirow{3}{*}{$\begin{array}{c}\text { Blood } \\
\text { acetaldehyde } \\
(\mathrm{nmol} / \mathrm{ml})\end{array}$} & \multirow{2}{*}{$\begin{array}{c}\text { Blood } \\
\text { ethanol } \\
\text { (mg/dl) }\end{array}$} \\
\hline & & & Low $K_{m}$ ALDH & High $K_{m}$ ALDH & & \\
\hline & & -- & $-\cdots-\cdots-$ & & & \\
\hline None (control) & 3 & & $15.14 \pm 1.69$ & $17.35 \pm 4.26$ & $-\ldots$ & 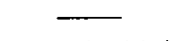 \\
\hline Ethanol & 4 & & $15.27 \pm 0.95$ & $16.41 \pm 0.49$ & $5.8 \pm 2.5$ & $145.6 \pm 11.0$ \\
\hline CMZ+Ethanol & 4 & & $7.41 \pm 0.40^{*}$ & $15.40 \pm 0.55$ & $65.4 \pm 6.1^{* *}$ & $176.4 \pm 17.5$ \\
\hline CPZ+Ethanol & 4 & & $5.96 \pm 0.61^{* *}$ & $16.05=0.53$ & $70.7 \pm 9.9^{* *}$ & $178.3 \pm 11.7$ \\
\hline LMOX+Ethanol & 4 & & $4.64 \pm 0.31^{* *}$ & $17.30 \pm 0.40$ & $95.9 \pm 4.3^{* *}$ & $185.8 \pm 6.6$ \\
\hline CEZ + Ethanol & 3 & & $14.32 \pm 0.76$ & $13.68 \pm 1.84$ & $10.2 \pm 0.9$ & $102.4 \pm 30.4$ \\
\hline CTM+Ethanol & 3 & & $13.73 \pm 1.29$ & $14.96 \pm 0.69$ & $11.6 \pm 2.0$ & $176.5 \pm 15.5$ \\
\hline
\end{tabular}

Animals were given a single subcutaneous administration of various antibiotics at $1.0 \mathrm{~g} / \mathrm{kg}$ and then oral administration of ethanol at $2.0 \mathrm{~g} / \mathrm{kg}$. Other treatments were the same as those in Table 1 . The values in the table represent the mean \pm S.E. Statistical analyses ( ${ }^{*} P<0.01$ ) in ALDH activity and in blood acetaldehyde level were performed against the control and ethanol-treated groups. respectively.

the slow disappearance or low metabolic capacity of alcohol in antibiotics-treated rats. However, no correlation was obtained between the increase in blood ethanol level and the depression of low $K_{m}$ enzyme activity; CTM-pretreatment caused no alterations in the low $K_{m} A L D H$ activity and blood acetaldehyde level, although the pretreated animals showed higher blood ethanol concentration (Table 2). In another experiment, germ-free rats were given a higher dose $(1.0 \mathrm{~g} / \mathrm{kg})$ of CMD for 3 days, and then ethanol was given 1 day after the last administration of CMD. A decrease in liver mitochondrial low $\mathrm{K}_{\mathrm{m}}$ ALDH activity and an increase in blood acetaldehyde level were observed in these animals as was observed in those given a single administration of CMD (data not shown).

\section{Discussion}

Disulfiram-like effects of beta-lactam antibiotics on the alcohol-metabolizing system were observed in conventional rats as a decrease in liver mitochondrial low $\mathrm{K}_{\mathrm{m}}$ ALDH activity (4, 5) and an increase in blood acetaldehyde level during ethanol metabolism (1-7). Beta-lactam antibiotics showing disulfiram-like effects have been shown to have NMTT as the $3^{\prime}$-position substituent of the cephalosporin nucleus $(1-6)$. The present report indicated that the NMTT-containing beta-lactam antibiotics (CMD, CMZ, CPZ and LMOX) had the disulfiram-like effects even in germ-free rats.

Previous reports demonstrated that the disulfiram-like effects, detected as the decrease in low $\mathrm{K}_{\mathrm{m}}$ ALDH activity and the increase in blood acetaldehyde level, were developed in rats by the administration of either the NMTT-containing antibiotics or NMTT itself (3-6), and the rapid onset of the effects was observed after the administration of NMTT rather than the NMTT-containing antibiotics $(10,11)$. When animals were given the antibiotics intravenously or subcutaneously, the disulfiram-like effects were observed following the disappearance of the drugs from the blood and liver $(9,10)$. After the oral administration of the NMTTcontaining antibiotics, higher concentrations of NMTT, but not its parent antibiotics, were detected in the blood and liver associated with the development of pronounced disulfiram-like effects (9). These results indicate clearly that the NMTT released from its parent antibiotics causes the development of the disulfiram-like effects. The present results (Table 1 ) obtained in germ-free rats are consistent with the above observations.

The biliary excretion of NMTT-containing antibiotics is closely correlated with the develoment of the disulfiram-like effects of antibiotics (7-9). The degradation of antibiotics and liberation of NMTT have been suggested to be bacteria-mediated reactions (8). However, the present results indicate that intestinal bacteria do not participate in 
releasing NMTT from the parent antibiotics. Recently, Uchida et al. (12) reported the decomposition of LMOX and the release of NMTT from the antibiotic in human and rat bile. Several investigators also reported the non-enzymatic decomposition of NMTTcontaining beta-lactam antibiotics in acidic and alkaline solution to release the free form of NMTT (13-16). All these findings indicate that the NMTT-containing beta-lactam antibiotics excreted into the bile are decomposed in the bile duct and the intestine under alkaline and acidic conditions, and the NMTT thus released is absorbed from the intestinal mucosa. Development of disulfiramlike effects even in the germ-free rats (Tables 1 and 2) supports the above assumption indicating no participation of intestinal bacteria to release NMT from its parent antibiotics. Nakamura et al. (17) reported the complete absorption of orally administered NMTT, which offers support for the above assumption. This causes NMTT to remain in the body at high levels for long periods, which leads to the production of the disulfiram-like effects. Therefore, an intestinal microfloral-mediated reaction does not seem to be the major factor in the development of the disulfiram-like effects of NMTT-containing beta-lactam antibiotics.

\section{References}

1 Buening, M.K., Wold, J.S., Israel, K.S. and Kammer, R.B.: Disulfiram-like reaction to betalactams. JAMA 245, 2027-2028 (1980)

2 Buening, M.K. and Wold, J.S.: Ethanolmoxalactam interactions in vivo. Rev. Infect. Dis. 4. Supp. S555-S563 (1982)

3 Yanagihara, M., Okada, K., Nozaki, M., Tsurumi, $\mathrm{K}$. and Fujimura, $\mathrm{H}$.: Cephem antibiotics and alcohol metabolism. (1) Disulfiram-like reaction resulting from intravenous administration of cephem antibiotics. Folia Pharmacol. Japon. 79, 551-560 (1982) (Abs. in English)

4 Yamanaka, Y., Yamamoto, T. and Egashira, T.: Effects of cephem antibiotics on rat liver aldehyde dehydrogenases. Japan. J. Pharmacol. 33, 717-723 (1983)

5 Matsubara, T., Otsubo, S., Ogawa, A., Okamoto, J., Yoshizaki, T., Nishibe, Y., Tochino, Y, and Hirai, E.: A comparative study on the effects of disulfiram and beta-lactam antibiotics on the acetaldehyde-metabolizing system in rats. Japan.
J. Pharmacol. 42, 333-343 (1986)

6 Nakahata, H., Hirai, Y., Kumasaka, Y., Miyazawa, T., Nakamura, $T$, and Imamura, $K_{\text {: }}$ : Disulfiram-like reactions resulting from administration of cephem antibiotics with methyltetrazolethiol moiety examined by using different doses. Japan. J. Antibiotics 39, 693-700 (1986) (Abs. in English)

7 Yanagihara, M., Okada, K., Nozaki, M., Tsurumi, $\mathrm{K}$, and Fujimura, $\mathrm{H}_{\text {.: }}$ Cephem antibiotics and alcohol metabolism. Japan. J. Antibiotics 38 , 634-642 (1985)

8 Turcan, R.G., MacDonald, C.M., Ings, R.M.J and Coombes, J.D.: Inhibition of the rate of ${ }^{74} \mathrm{CO}_{2}$ production from $\left[{ }^{14} \mathrm{C}\right]$ ethanol in rats given beta-laciam antibiotics with disulfiram. like effects. Antimicrob. Agents Chemother. 27, 535-540 (1985)

9 Matsubara, T., Otsubo, S., Ogawa, A. and Oka, T.: Pharmacokinetics of latamoxef and $\mathrm{N}$ methyltetrazolethiol in rats associated with the development of disulfiram-like effects. Japan. J. Pharmacol. 45 (1987) (in press)

10 Matsubara, T., Otsubo, S., Ogawa, A., Kawamoto, K., Okamoto, J., Sugeno, K, Tochino, Y., Yoshida, T. and Hirai, E.: Effects of beta-lactam antibiotics and $\mathrm{N}$-methyltetrazolethiol on the alcohol-metabolizing system in rats. Japan. J. Pharmacol. 45 (1987) (in press)

11 Brien, J.F., Tam. G.S., Cameron, R.J., Steenaart, N.A.E. and Loomis, C.W: A comparative study of the inhibition of hepatic aldehyde dehydrogenases in the rat by methyltetrazolethiol, calcium carbimide, and disulfiram. Can. J. Physiol. Pharmacol. 63, 438-443 (1985)

12 Uchida, K., Konishi, M., Akiyoshi, T., Igimi, H. and Asakawa, S.: Biliary excretion of latamoxef and $\mathrm{N}$-methyltetrazolethiol in humans and rats. J. Pharmacobiodyn. 8, 981-988 (1985)

13 Saikawa, I., Momonori, K., Sakai, H., Takashita,

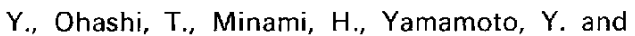
Fukuoka, $Y$ :: Studies on beta-lactam antibiotics for medicinal purpose. $X$. Yakugaku Zasshi 99 , 1207-1218 (1979) (Abs. in English)

14 Minor, D.J., Coleman, D.L., Shepherd, A.M.M. and Hardin, T.C.: Determination of moxalactam in human body fluids by liquid chromatographic and microbiological methods. Antimicrob. Agents Chemother. 20, 252-257 (1981)

15 Wise, R, and Dent, J.: Stability of beta-lactam. antibiotics containing $\mathrm{N}$-methyithiotetrazole side-chain. Lancet ii, 624-625 (1983)

16 Hashimoto, N., Tasaki, T. and Tanaka, H.: Degradation and epimerization kinetics of moxalactam in aqueous solution. J. Pharm. Sci. 
$73 \quad 369-373$ (1984)

17 Nakamura K., Nakagawa, A., Tanaka, M., Masuda, $H_{\text {, }}$, Hayashi, $Y$. and Saionji, K.: Effects of cephem antibiatics on ethanol metabolism Folıa Pharmacol. Japon. 83 183-191 (1984) (Abs: in English) 